\title{
A Patient With Intermittent Abdominal Pain for Three Weeks
}

\section{Hyun Seok Lee}

Department of Internal Medicine, Kyungpook National University School of Medicine, Daegu, Korea
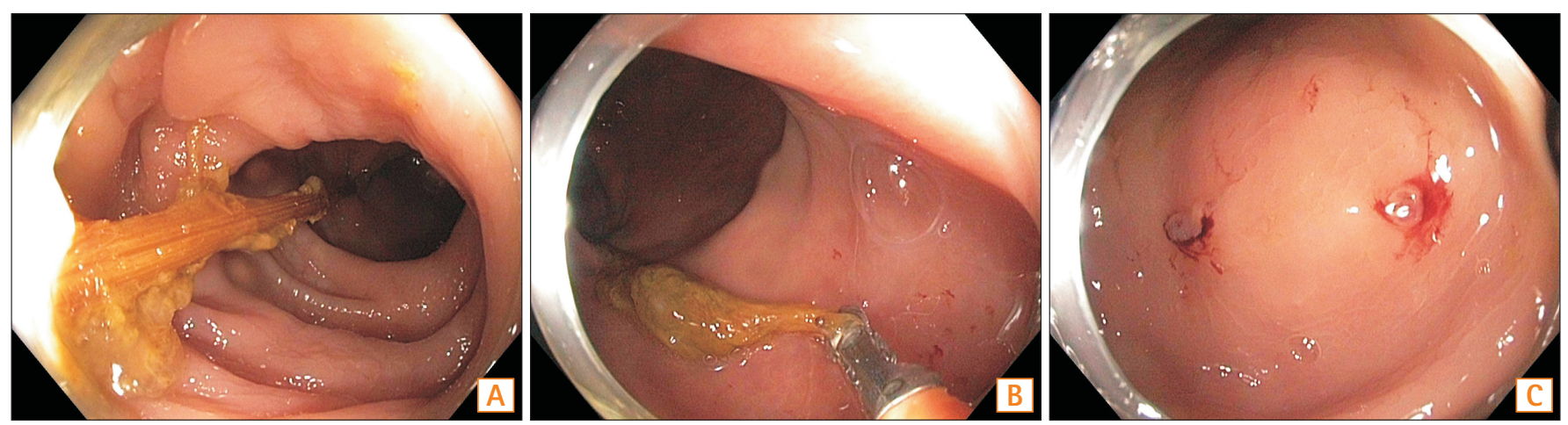

Question: A 52-year-old woman visited our hospital complaining of lower abdominal pain and intermittent chilly sensations for three weeks. She had taken over-the-counter remedies for a cold three weeks prior to her visit. As the symptoms persisted, she visited another clinic where she underwent an esophagogastroduodenoscopy and colonoscopy. The esophagogastroduodenoscopy did not reveal any possible cause of her abdominal pain. Colonoscopy revealed a long and thin foreign body perforating her sigmoid colon, and this led to her being admitted to the outpatient clinic of our hospital.

Her medical history involved no serious illness or surgical procedure besides hypertension. We performed a sigmoidoscopy to evaluate and remove the foreign body. This revealed a hard, long, and thin foreign body lodged in the

\footnotetext{
Received December 7, 2014. Accepted December 8, 2014. Correspondence to Hyun Seok Lee, Department of Internal Medicine, Kyungpook National University Medical Center, Kyungpook National University School of Medicine, 807 Hoguk-ro, Buk-gu, Daegu 702-210, Korea. Tel: +82-53-200-2603, Fax: +82-53-200-2027, E-mail: Ihsworld@ nate.com

Financial support: None. Conflict of interest: None.
}

sigmoid colon, with both ends impacted into the bowel wall (Fig. A). The end of the foreign body was carefully and firmly grasped with forceps, and gently dislodged from the colon wall (Fig. B). It was easily removed intact through the anus, and the procedure was uneventful. Focal mucosal edema with bloody erythema was noted at the sigmoid colon after removing the foreign body (Fig. C). Her abdominal pain improved considerably immediately after removal.

The post-procedure abdominal radiograph was negative for pneumoperitoneum. An abdominal CT scan was performed, and revealed a small amount of extraluminal air with surrounding fluid at the sigmoid mesocolon, and shortsegmental sigmoid colon edema. Treatment with intravenous broad-spectrum antibiotics was initiated while she remained fasted. A regular diet was introduced gradually after five days of fasting. During this period, she noted complete resolution of her lower abdominal pain and chilly sensations. What is the most likely diagnosis?

๑ Copyright 2015. Korean Association for the Study of Intestinal Diseases. All rights reserved.

This is an Open Access article distributed under the terms of the Creative Commons Attribution Non-Commercial License (http://creativecommons.org/licenses/by-nc/3.0) which permits unrestricted non-commercial use, distribution, and reproduction in any medium, provided the original work is properly cited. 

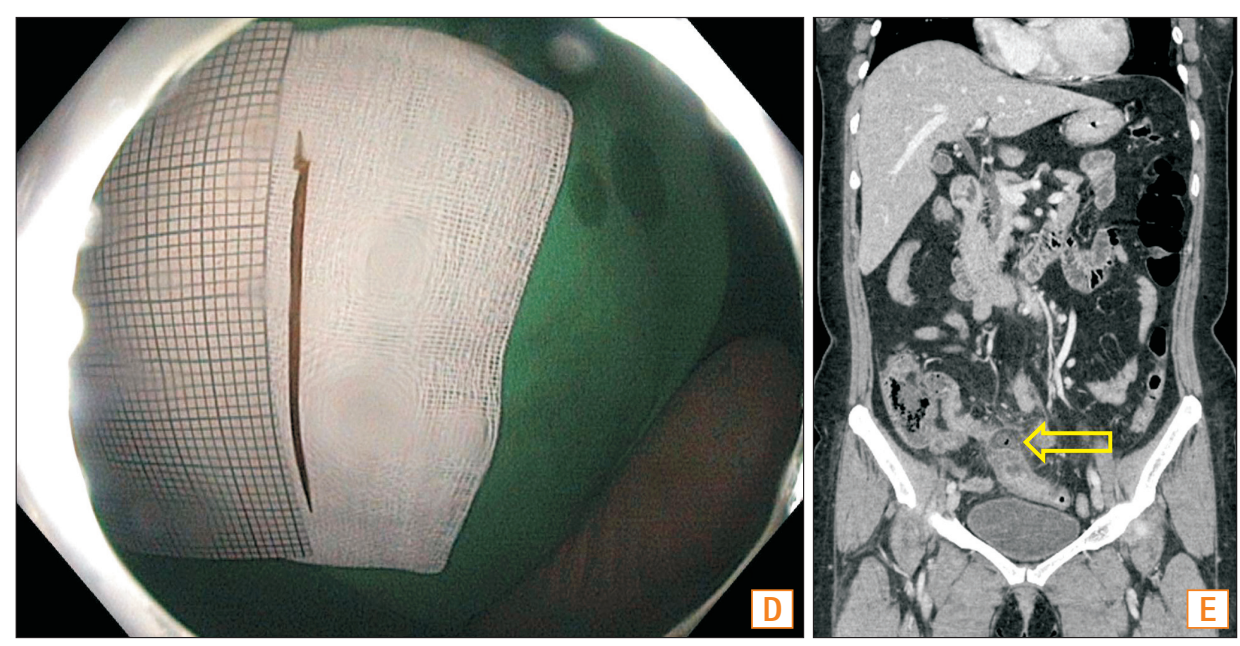

\section{Answer to the Images: A Toothpick Perforating the Sigmoid Colon}

The ingestion of foreign bodies is a common clinical problem. Large, thin, and sharp objects (toothpicks, chicken or fish bones, and metal objects) carry a high risk of bowel perforation. ${ }^{1}$ Swallowed objects larger than $65 \mathrm{~mm}$ in length that pass beyond the esophagogastric junction are likely to cause complications and require surgical intervention. ${ }^{2}$ Toothpick ingestion is commonly implicated in bowel injuries due the two sharply pointed ends and indigestible hard body of the object, which make it difficult for the toothpick to pass the intestinal lumen, especially in the narrow or tortuous sections of the gastrointestinal tract or at the transition from a mobile portion of the bowel (ileum and sigmoid colon) to a more fixed portion (cecum and rectum). ${ }^{3}$

In this case, a healthy woman presented with intermittent lower abdominal pain that started a few days after the accidental ingestion of a toothpick. She had no psychiatric problems, normal teeth, and had not been drinking alcohol at the time. After she was informed that the foreign body was a toothpick (Fig. D), she remembered eating with the aid of a toothpick three weeks prior. However, she did not experience any symptoms while swallowing the toothpick, nor did she recall ingesting it.
After removing the toothpick, an abdominal CT scan revealed microperforation at the sigmoid colon (Fig. E). Her symptoms were improved by conservative treatment over several days. Intestinal perforation due to foreign body ingestion should be considered in the differential diagnosis of patients presenting with abdominal discomfort or abdominal pain, even when they do not recall any foreign body ingestion. ${ }^{4}$

\section{REFERENCES}

1. Velitchkov NG, Grigorov GI, Losanoff JE, Kjossev KT. Ingested foreign bodies of the gastrointestinal tract: retrospective analysis of 542 cases. World J Surg 1996;20:1001-1005.

2. Gracia C, Frey CF, Bodai BI. Diagnosis and management of ingested foreign bodies: a ten-year experience. Ann Emerg Med 1984;13:30-34.

3. Pinero Madrona A, Fernandez Hernandez JA, Carrasco Prats M, Riquelme Riquelme J, Parrila Paricio P. Intestinal perforation by foreign bodies. Eur J Surg 2000;166:307-309.

4. Zezos P, Oikonomou A, Souftas V, Gkotsis D, Pitiakoudis M, Kouklakis G. Endoscopic removal of a toothpick perforating the sigmoid colon and causing chronic abdominal pain: a case report. Cases J 2009;2:8469. 